\title{
Quantitative assessment of the association between glutathione S-transferase M1 polymorphism and the risk of developing nasopharyngeal cancer
}

\author{
YAN ZHAO, YUBAO WANG, XIAOMEI WU, JUN WANG, LIJUAN ZHANG, YUCHI JIA and WEI QI \\ Tianjin Institute of Infectious Diseases, The Second Hospital of Tianjin Medical University, Tianjin 300211, P.R. China
}

Received October 1, 2014; Accepted July 9, 2015

DOI: $10.3892 / \mathrm{ol} .2015 .3848$

\begin{abstract}
Glutathione S-transferases (GSTs) participate in the detoxification and elimination of electrophilic carcinogens by conjugating them to glutathione. Previous studies have reported a potential association between GSTM1 polymorphism and the risk of developing nasopharyngeal cancer (NPC). However, those findings remain controversial. In the present study, a meta-analysis was conducted by pooling the odds ratios (ORs) with the corresponding 95\% confidence intervals (CIs) of all the available case-control studies on NPC. A comprehensive search of PubMed, Embase, Web of Science and China National Knowledge Infrastructure databases up to May $13^{\text {th }}, 2014$ was performed to identify eligible studies. A total of 12 separate publications, involving 1,593 cases of NPC and 2,868 controls, were included in the meta-analysis. The results demonstrated that the null genotype of GSTM1 was significantly associated with increased risk of developing NPC $\left(\mathrm{OR}=1.530,95 \% \mathrm{CI}=1.348-1.737, \mathrm{P}_{\text {heterogeneity }}=0.370\right)$. Subgroup analysis by ethnicity suggested that Asian carriers of the GSTM1 null genotype were more susceptible to NPC than individuals from other ethnic groups $(\mathrm{OR}=1.516$, $\left.95 \% \mathrm{CI}=1.328-1.731, \mathrm{P}_{\text {heterogeneity }}=0.270\right)$. Sensitivity analysis confirmed the stability of these observations. In conclusion, the results from the present meta-analysis indicated that the GSTM1 polymorphism may be involved in the development of NPC, particularly in Asians.
\end{abstract}

\section{Introduction}

Nasopharyngeal carcinoma (NPC) is a rare condition worldwide, but it is endemic in certain populations (1). The development of NPC may be attributed to the dynamic interplay

Correspondence to: Professor Wei Qi, Tianjin Institute of Infectious Diseases, The Second Hospital of Tianjin Medical University, 23 Pingjiang Road, Hexi, Tianjin 300211, P.R. China E-mail: weiqi-gryjs@163.com

Key words: nasopharyngeal cancer, glutathione S-transferase M1, polymorphisms, meta-analysis of environmental factors and genetic susceptibility. Host factors, including tobacco smoking, consumption of salt-preserved fish, history of chronic respiratory tract diseases and Epstein-Barr virus (EBV) infection, are well-established risk factors for the development of NPC $(2,3)$. However, not all individuals exposed to EBV infection and environmental carcinogenic factors develop NPC, which indicates that genetic susceptibility may also contribute. Glutathione S-transferases (GSTs) are a large family of phase II detoxification enzymes that regulate the conversion of toxic compounds to hydrophilic metabolites $(4,5)$. GSTM1 is one of the main subtypes of GSTs, and is involved in protecting hosts against cancer (4). The GSTM1 gene is located on the short arm of chromosome 1 (1p13.3), and displays several polymorphisms (6). The most common polymorphism in the GSTM1 gene is a null variant, which has been widely investigated as a risk biomarker for various types of cancer $(7,8)$. The GSTM1 null variant may lead to the absence of enzymatic activity, and individuals who carry this variant are thought to be at increased risk of developing cancer (6). Numerous studies have previously evaluated the association of the GSTM1 polymorphism with the susceptibility to NPC, but the results are inconsistent, possibly due to the limited sample size and low power of these studies, which are insufficient to detect the precise effect in a single study (9-23). Thus, in the present study, a meta-analysis was performed in order to quantitatively evaluate the association between the GSTM1 polymorphism and the susceptibility to NPC.

\section{Materials and methods}

Search strategy. A comprehensive search of PubMed, Embase, Web of Science and China National Knowledge Infrastructure (CNKI) databases was performed up to May $13^{\text {th }}, 2014$, in order to identify potentially relevant studies on the association between the GSTM1 polymorphism and the risk of developing NPC. The literature search was conducted independently by 2 investigators, and the disagreements were resolved by consensus. The search terms were as follows: Glutathione S-transferase, GST, GSTs, glutathione S-transferase M1, GSTM, GSTM1, polymorphism, mutation or variation; with nasopharyngeal cancer, nasopharyngeal carcinoma or NPC. A manual search of the references of all the retrieved publications was conducted to identify additional studies. There were no language or sample size limitations. 


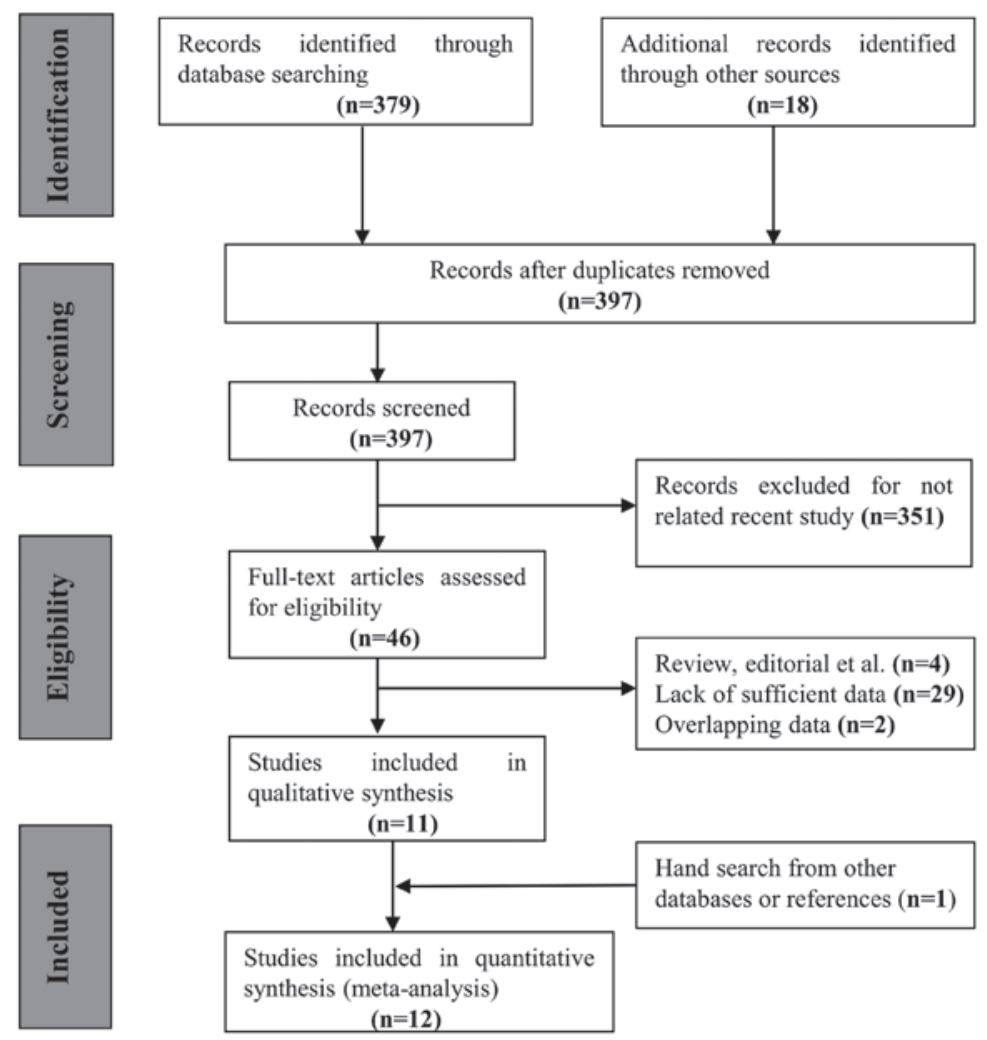

Figure 1. Flow diagram of the selection process followed for identifying and selecting eligible studies for the present meta-analysis.

Inclusion and exclusion criteria. Studies were included in the present meta-analysis according to the following inclusion criteria: i) Studies that evaluated the association between the GSTM1 polymorphism and the risk of developing NPC; ii) those that were case-control studies; iii) those that described the diagnoses of NPC and the sources of cases and controls; iv) those that contained available data for acquiring numbers of the null and present genotypes of GSTM1; and iv) those that presented sufficient information for the calculation of the odds ratio (OR) with its corresponding 95\% confidence interval (CI). Single cases and family-based studies were excluded from the meta-analysis. In those cases of overlapping studies, the latest or the most complete one was selected for inclusion in the analysis.

Data extraction. The following data were extracted from the relevant publications: First author's name; year of publication; ethnicity and country of origin of cohort; numbers of cases and controls; genotyping method; source of controls; and genotype distributions in cases and controls. Disagreements were settled through discussion among the 2 investigators mentioned above.

Statistical analysis. The strength of the associations between the GSTM1 polymorphism and the risk of developing NPC was estimated by OR and the corresponding 95\% CI, based on the frequencies of the null and present genotypes in cases and controls. The pooled ORs for the null vs. present genotype were calculated using the fixed- or random-effects model (known as the Mantel-Haenszel and DerSimonian and Laird method, respectively), and determined by the $\mathrm{Z}$ test $(24,25)$.
$\mathrm{Q}$ and $\mathrm{I}^{2}$ tests were adopted to assess the heterogeneity among the studies included in the meta-analysis. $\mathrm{P}<0.01$ and $\mathrm{I}^{2}>50 \%$ were considered to indicate a statistically significant difference in heterogeneity $(26,27)$. Subgroup analyses were also conducted according to ethnicity: Of the 12 included studies, 10 were conducted in Asians (11-16,18,20-22), 1 in North Africans (17) and 1 in a mixed population (9). Publication bias was evaluated by funnel plot (28) and Egger's linear regression test (29). Sensitivity analysis by omission of each individual study was performed to identify the source of between-study heterogeneity and to confirm the stability and reliability of the pooled results (30). The statistical analyses were performed using STATA12.0 software (StataCorp LP, College Station, TX, USA).

\section{Results}

Study characteristics. Relevant studies were retrieved following a systematic literature search of PubMed, Embase, Web of Science and CNKI databases. The selection process for the articles included in the present meta-analysis is depicted in Fig. 1. The initial search yielded 394 study titles and abstracts, which were then subjected to an independent review. Based on the inclusion criteria, 12 individual publications studying the association of the GSTM1 polymorphism and the risk of developing NPC in a total of 1,593 cases and 2,868 individuals were available for analysis (9,11-17,20-22). The clinical characteristics of these studies are listed in Table I. Among the 12 included studies, 10 were conducted in Asians (11-16,18,20-22), 1 in North African (17) and 1 in a mixed population (9). 
Table I. Summary of the main characteristics of the studies included in the present meta-analysis.

\begin{tabular}{|c|c|c|c|c|c|c|c|c|c|}
\hline \multirow[b]{3}{*}{ First author } & \multirow[b]{3}{*}{ Ref. } & \multirow{2}{*}{\multicolumn{2}{|c|}{ Cohort }} & \multirow{2}{*}{\multicolumn{2}{|c|}{ Sample size }} & \multicolumn{4}{|c|}{ GSTM1 polymorphism } \\
\hline & & & & & & \multicolumn{2}{|c|}{ Null } & \multicolumn{2}{|c|}{ Present } \\
\hline & & Country & Ethnicity & Case & Control & Case & Control & Case & Control \\
\hline Nazar-Stewart V & 9 & USA & Mixed & 83 & 142 & 45 & 63 & 38 & 79 \\
\hline Da SJ & 11 & China & Asian & 80 & 80 & 48 & 36 & 32 & 44 \\
\hline Cheng YJ & 12 & Taiwan & Asian & 314 & 337 & 173 & 169 & 141 & 168 \\
\hline Liu ZG & 13 & China & Asian & 46 & 53 & 28 & 18 & 18 & 35 \\
\hline Deng ZL & 14 & China & Asian & 91 & 135 & 56 & 64 & 35 & 71 \\
\hline Deng ZL & 15 & China & Asian & 127 & 207 & 78 & 95 & 49 & 112 \\
\hline Tiwawech D & 16 & Thailand & Asian & 78 & 145 & 50 & 74 & 28 & 71 \\
\hline Bendjemana K & 17 & Tunisia & African & 45 & 100 & 23 & 33 & 22 & 67 \\
\hline Guo X & 18 & China & Asian & 341 & 590 & 204 & 328 & 137 & 262 \\
\hline Jiang Y & 20 & China & Asian & 182 & 366 & 97 & 157 & 85 & 215 \\
\hline Wei YP & 21 & China & Asian & 126 & 641 & 78 & 305 & 48 & 336 \\
\hline Liao ZL & 22 & China & Asian & 80 & 72 & 50 & 32 & 30 & 40 \\
\hline
\end{tabular}

GSTM1, glutathione S-transferase M1.

\begin{tabular}{|c|c|c|c|}
\hline Study ID & Ref. & OR $(95 \% \mathrm{Cl})$ & Weight $(\%)$ \\
\hline Nazar-Stewart V & 9 & $1.48(0.86,2.56)$ & 5.50 \\
\hline Da SJ & 11 & $1.83(0.98,3.43)$ & 3.72 \\
\hline Cheng YJ & 12 & $1.22(0.90,1.66)$ & 18.90 \\
\hline Liu ZG & 13 & $3.02(1.33,6.87)$ & 1.69 \\
\hline Deng ZL & 14 & $1.77(1.03,3.05)$ & 5.12 \\
\hline Deng ZL & 15 & $1.88(1.20,2.94)$ & 7.20 \\
\hline Tiwawech D & 16 & $1.71(0.97,3.02)$ & 4.80 \\
\hline Bendjemana K & 17 & $2.12(1.04,4.35)$ & 2.59 \\
\hline Guo X & 19 & $1.19(0.91,1.56)$ & 24.93 \\
\hline Jiang $Y$ & 20 & $1.56(1.09,2.23)$ & 12.44 \\
\hline Wei YP & 21 & $1.79(1.21,2.65)$ & 9.86 \\
\hline Liao ZL & 22 & $2.08(1.09,3.99)$ & 3.26 \\
\hline Overall $(\mathrm{l}$-squared $=7.7 \%, \mathrm{P}=0.370$ ) & & $1.53(1.35,1.74)$ & 100.00 \\
\hline $\begin{array}{c}1 \\
0.145\end{array}$ & & & \\
\hline
\end{tabular}

Figure 2. Forest plots describing the associations between the glutathione S-transferase M1 polymorphism and the risk of developing nasopharyngeal cancer. $\mathrm{OR}$, odds ratio; $\mathrm{CI}$, confidence interval.

Pooled analysis results. Estimation of the association between the GSTM1 polymorphism and the susceptibility to NPC was performed, and the pooled OR for the GSTM1 polymorphism suggested a significantly increased risk of developing
NPC for carriers of the null genotype, compared with the present genotype $\left(\mathrm{OR}=1.530,95 \% \mathrm{CI}=1.348-1.737, \mathrm{P}_{\text {heteroge- }}\right.$ neity $=0.370$; Fig. 2). Sensitivity analysis by omission of each individual study further confirmed this significant association 


\section{Study ID Ref. Study ommited}

$\begin{array}{rr}\text { Nazar-Stewart V } & 9 \\ \text { Da SJ } & 11 \\ \text { Cheng YJ } & 12 \\ \text { Liu ZG } & 13 \\ \text { Deng ZL } & 14 \\ \text { Deng ZL } & 15 \\ \text { Tiwawech D } & 16 \\ \text { Bendjemana K } & 17 \\ \text { Guo X } & 19 \\ \text { Jiang Y } & 20 \\ \text { Wei YP } & 21 \\ \text { Liao ZL } & 22\end{array}$

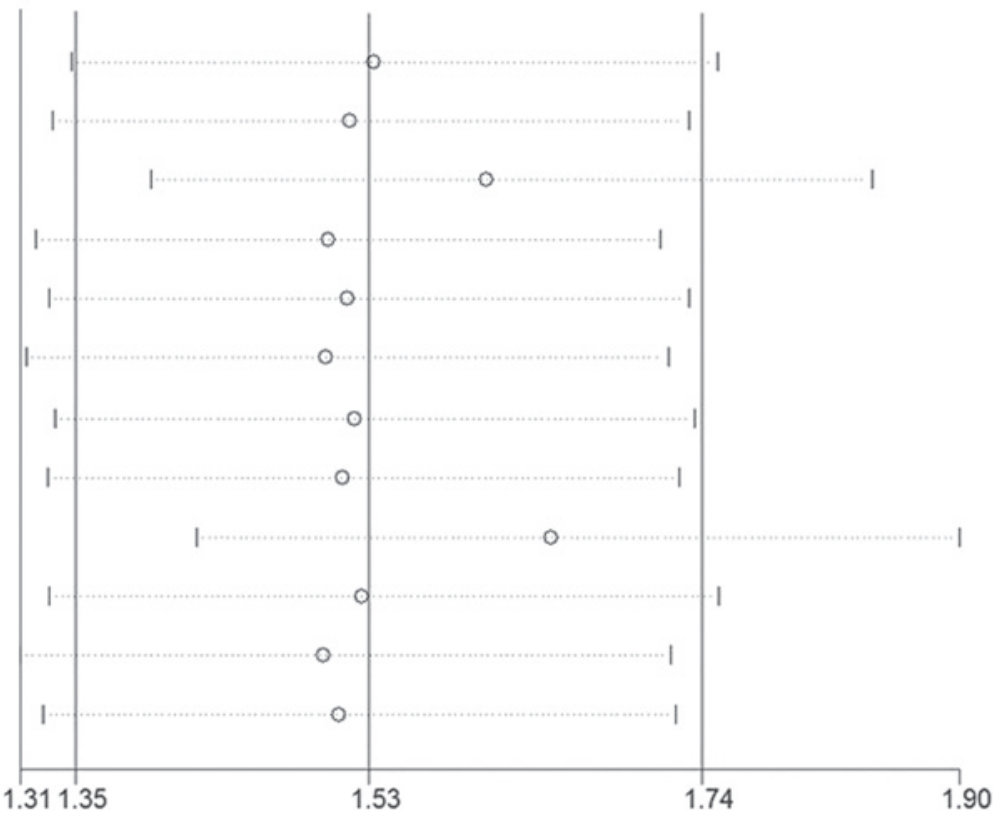

Figure 3. Sensitive analysis of the association between the glutathione S-transferase M1 null genotype and the risk of developing nasopharyngeal cancer.

(Fig. 3). In the subgroup analyses by ethnicity, a significantly increased risk of developing NPC was observed for the polymorphism of GSTM1 in the Asian population $(\mathrm{OR}=1.516$, 95\% CI=1.328-1.731, $\mathrm{P}_{\text {heterogeneity }}=0.270$ ). Subgroup analyses in the North African and mixed populations were not performed due to insufficient data availability.

Publication bias. Begg's funnel plot and Egger's test were used to estimate the publication bias of all the studies on the associations of the GSTM1 polymorphism and the risk of developing NPC that were included in the present meta-analysis. The funnel plot shapes of the Begg's test did not reveal any evidence of asymmetry (Fig. 4). Furthermore, the P-value of the Egger's test was $<0.05$, indicating absence of publication bias among the included studies (Fig. 5).

\section{Discussion}

GSTs belong to the biotransformation family of enzymes. GSTs are phase II enzymes with catalytic and noncatalytic activities in vivo, which are involved in the detoxification of electrophilic compounds by glutathione conjugation, including carcinogens and cytotoxic drugs $(31,32)$. Since the presumed function of GSTs is to protect tissues against toxic and carcinogenic compounds, they are considered to be important determinants in the development of prostate cancer. GSTM1, one of the main subtypes of GSTs, participates in the protection of the host against cancer (4). The GSTM1 gene displays several polymorphisms, among which, the null variant is the most common one, and it has been widely investigated as a risk biomarker for various types of cancer $(7,8)$. The GSTM1 null variant may result in the absence of enzymatic activity, and individuals who carry the null variant are thought to be at increased risk of developing cancer, since the polymorphic deletions of GSTs may affect their ability to detoxify electrophilic carcinogens, which may lead to an increase in the host's susceptibility to environmental toxins and carcinogens $(6,33)$. Mutations in GSTM1 are the most commonly studied polymorphisms of GSTs, regarding genetic susceptibility to cancer (34-36). Numerous individual case-control studies on the GSTM1 polymorphism in association with the risk of developing NPC have been conducted in the past 2 decades (9-23). Certain studies have previously suggested that the GSTM1 null genotype was associated with increased risk of developing NPC $(9,20)$, while other studies did not observe any significant associations between various genetic polymorphisms of GSTM1 and the risk of developing NPC (12).

These discrepancies may arise from the small size of the sample in individual case-control studies, which may result in insufficient statistical power, particularly for weak correlations. In addition, differences in genetic background, study design and source of cases and controls may also contribute to these controversial and inconclusive findings. Therefore, in the present study, a meta-analysis was conducted by pooling the ORs with $95 \%$ CIs of all the currently available case-control studies on GSTM1 and NPC, in order to clarify these apparently contradictory findings. A total of 12 eligible studies with 1,593 cases and 2,868 controls were analyzed to quantitatively evaluate the association between the GSTM1 polymorphism and the susceptibility to NPC. The results suggested that the GSTM1 null genotype was significantly associated with the risk of developing NPC. 


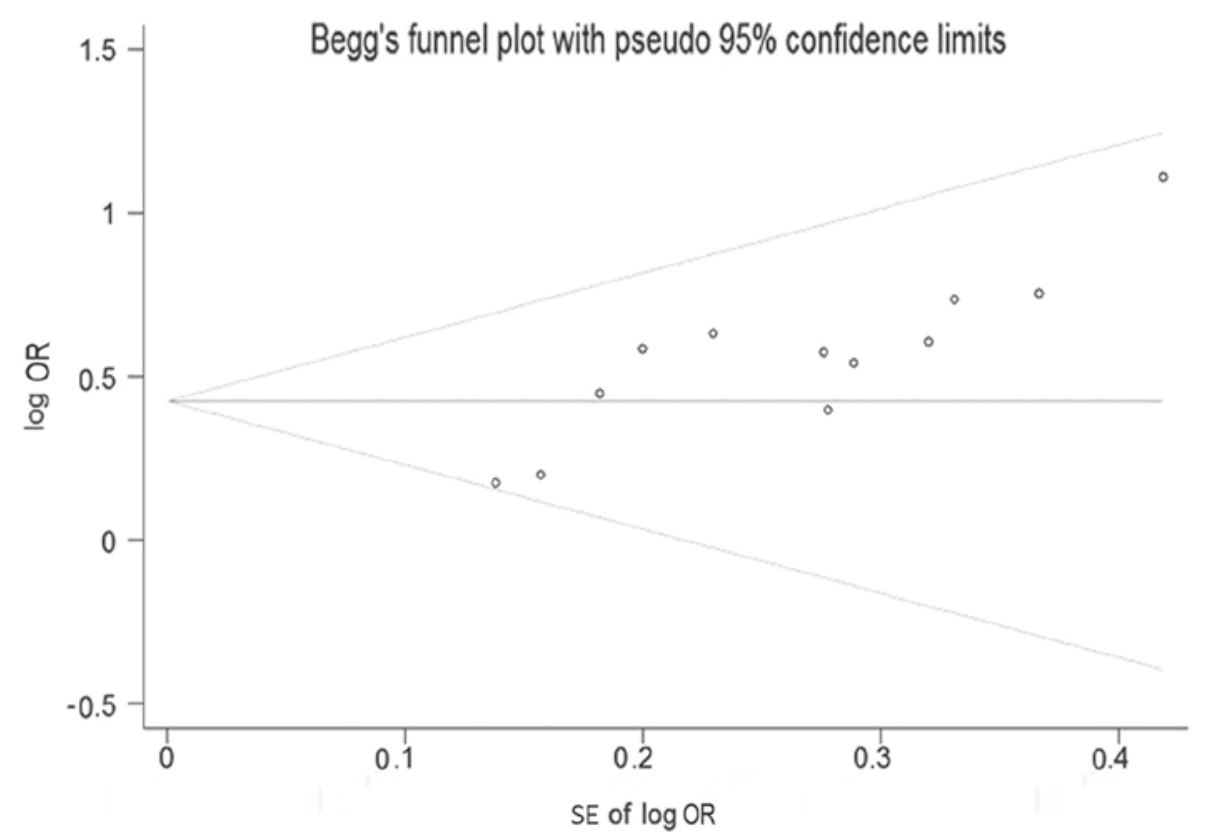

Figure 4. Begg's funnel plot for the risk of publication bias. OR, odds ratio; SE, standard error.

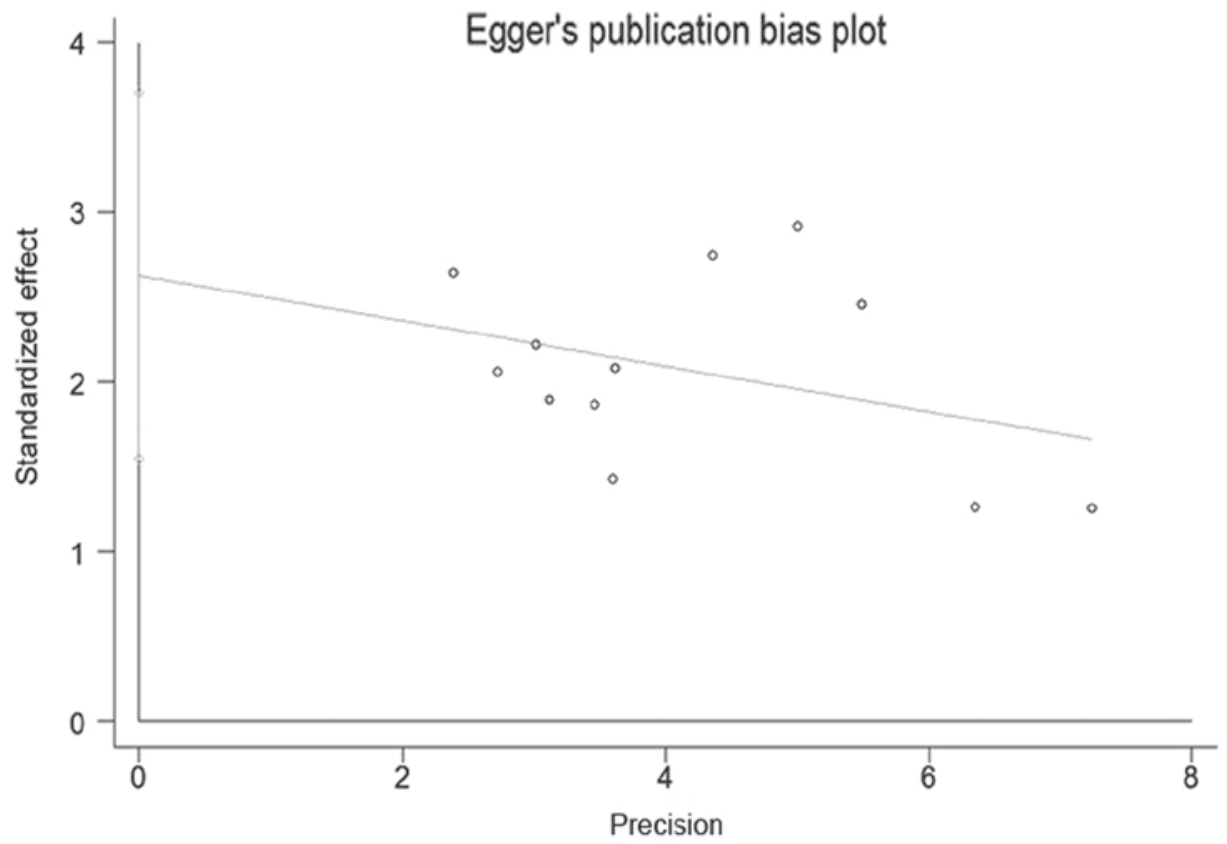

Figure 5. Egger's funnel plot for the risk of publication bias.

However, there were several limitations in the present meta-analysis: i) The adjusted estimation, which is a more precise way of estimating the association, was not pooled; thus, further studies are required to calculate the pooled OR, adjusting for other confounding factors; ii) a potential source of bias in studies of genotypes may be due to the inclusion of individuals from different ethnic backgrounds; and iii) gene-gene and gene-environment interactions were not analyzed in the present meta-analysis due to the unavailability of relevant studies. Therefore, additional case-control studies are required to further analyze these potential interactions and their role in the association between GSTM1 and NCP.
In summary, the results from the present meta-analysis indicate that the GSTM1 polymorphism participates in the development of NPC. However, the significant associations of the GSTM1 polymorphism with the risk of developing NPC must be validated in future studies, which must also account for the influence of gene-gene and gene-environment interactions in this potential association.

\section{Acknowledgements}

The authors would like to thank Dr Qiliang Cai (Department of Urology, The Second Hospital of Tianjin Medical University, 
Tianjin Institute of Urology, Tianjin, China) for the invaluable discussions regarding study design and statistical analyses.

\section{References}

1. Parkin DM, Bray F, Ferlay J and Pisani P: Global cancer statistics, 2002. CA Cancer J Clin 55: 74-108, 2005.

2. Chang ET and Adami HO: The enigmatic epidemiology of nasopharyngeal carcinoma. Cancer Epidemiol Biomarkers Prev 15: $1765-1777,2006$.

3. O'Neil JD, Owen TJ, Wood VH, Date KL, Valentine R, Chukwuma MB. Arrand JR, Dawson CW and Young LS Epstein-Barr virus-encoded EBNA1 modulates the AP-1 transcription factor pathway in nasopharyngeal carcinoma cells and enhances angiogenesis in vitro. J Gen Virol 89: 2833- 2842, 2008

4. Strange RC, Spiteri MA, Ramachandran S and Fryer AA Glutathione-S-transferase family of enzymes. Mutat Res 482: 21-26, 2001.

5. Oakley A: Glutathione transferases: A structural perspective. Drug Metab Rev 43: 138-151, 2011.

6. Hayes JD and Strange RC: Glutathione S-transferase polymorphisms and their biological consequences. Pharmacology 61: $154-166,2000$.

7. Zhang ZJ, Hao K, Shi R, Zhao G, Jiang GX, Song Y, Xu X and Ma J: Glutathione S-transferase M1 (GSTM1) and glutathione S-transferase T1 (GSTT1) null polymorphisms, smoking, and their interaction in oral cancer: A HuGE review and meta-analysis. Am J Epidemiol 173: 847-857, 2011.

8. Taioli E, Flores-Obando RE, Agalliu I, Blanchet P, Bunker CH, Ferrell RE, Jackson M, Kidd LC, Kolb S, Lavender NA, et al: Multi-institutional prostate cancer study of genetic susceptibility in populations of African descent. Carcinogenesis 32: $1361-1365,2011$.

9. Nazar-Stewart V, Vaughan TL, Burt RD, Chen C, Berwick M and Swanson GM: Glutathione S-transferase M1 and susceptibility to nasopharyngeal carcinoma. Cancer Epidemiol Biomarkers Prev 8: 547-551, 1999.

10. Guo XL: Genetic polymorphism of cytochrome P450 1A1 and glutathione S-transferase Ml related with susceptibility of nasopharyngeal carcinoma. Hebei Med Univ. 2001.

11. Da SJ, Liang B, Wu HL and Guan LL: Relationship between GSTM1 gene polymorphism and genetic susceptibility in nasopharyngeal carcinoma. Prac J of Cancer 17: 617-619, 2002 (In Chinese)

12. Cheng YJ, Chien YC, Hildesheim A, Hsu MM, Chen IH, Chuang J, Chang J, Ma YD, Luo CT, Hsu WL, et al: No association between genetic polymorphisms of CYP1A1, GSTM1, GSTT1, GSTP1, NAT2, and nasopharyngeal carcinoma in Taiwan. Cancer Epidemiol Biomarkers Prev 12: 179-180, 2003.

13. Liu ZG, Wei YP and Ma Y: Population with GSTT1 gene deletion and the relationship to hepatocellular carcinoma from Guangxi. J Guangxi Med Univ 20: 161-163, 2003. (In Chinese).

14. Deng ZL, Wei YP and Ma Y: Frequent genetic deletion of detoxifying enzyme GSTM1 and GSTT1 genes in nasopharyngeal carcinoma patients in Guangxi Province, China. Zhonghua Zhong Liu Za Zhi 26: 598- 600, 2004 (In Chinese).

15. Deng ZL, Wei YP, Luo W, Liao ZL and Ma Y: Glutathione S-transferase M1 and T1 gene deletion associated with increased susceptibility to nasopharyngeal carcinoma. Chinese-German J Clin Oncol 4: 276-278, 2005.

16. Tiwawech D, Srivatanakul P, Karalak A and Ishida T: Glutathione S-transferase M1 gene polymorphism in Thai nasopharyngeal carcinoma. Asian Pac J Cancer Prev 6: 270-275, 2005.

17. Bendjemana K, Abdennebi M, Gara S, Jmal A, Ghanem A, Touati S, Boussen H, Ladgham A and Guemira F: Genetic polymorphism of gluthation-S transferases and $\mathrm{N}$-acetyl transferases 2 and nasopharyngeal carcinoma: The Tunisia experience. Bull Cancer 93: 297-302, 2006.
18. Guo X, O'Brien SJ, Zeng Y, Nelson GW and Winkler CA: GSTM1 and GSTT1 gene deletions and the risk for nasopharyngeal carcinoma in Han Chinese. Cancer Epidemiol Biomarkers Prev 17: 1760-1763, 2008.

19. He Y, Zhou GQ, Li X, Dong XJ, Chai XQ and Yao KT: Correlation of polymorphism of the coding region of glutathione S-transferase M1 to susceptibility of nasopharyngeal carcinoma in South China population. Ai Zheng 28: 5-7, 2009 (In Chinese).

20. Jiang Y, Li N, Dong P, Zhang N, Sun Y, Han M, Wen J and Chen M: Polymorphisms in GSTM1, GSTTI and GSTP1 and nasopharyngeal cancer in the East of China: a case-control study. Asian Pacif J Cancer Prev 12: 3097-3100, 2011.

21. Wei YP, Long XD, Liu ZG, Ma Ya and Deng ZL: Genetic polymorphism of glutathione-S-transferase M1 and T1 in associated with carcinogenesis of hepatocellular carcinoma and nasopharyngeal carcinoma. Chinese-German J Clin Oncol 11: 138-141, 2012 .

22. Liao ZL, Deng ZL, Wei YP, Xie KS, Zhang B, Dai XM and $\mathrm{Xu}$ CS: Relationship of GSTT1 and GSTM1 gene polymorphisms with the development of nasopharyngeal carcinoma. J Guangxi Med Univ 22: 372-374, 2005.

23. Tian SZ: The analysis of clinical factors related to laryngeal carcinoma and research on the association between genetic polymorphisms of xenobiotic-metabolizing enzymes, DNA repair genes and susceptibility to laryngeal carcinoma. Zhongshan Univ 2001

24. DerSimonian R and Laird N: Meta-analysis in clinical trials. Control Clin Trials 7: 177-188, 1986.

25. Mantel N and Haenszel W: Statistical aspects of the analysis of data from retrospective studies of disease. J Natl Cancer Inst 22: 719-748, 1959.

26. Cochran WG: The comparison of percentages in matched samples. Biometrika 37: 256-266, 1950.

27. Higgins JP, Thompson SG, Deeks JJ and Altman DG: Measuring inconsistency in meta-analyses. BMJ 327: 557-560, 2003

28. Stuck AE, Rubenstein LZ and Wieland D: Bias in meta-analysis detected by a simple, graphical test: Asymmetry detected in funnel plot was probably due to true heterogeneity. BMJ 316 : 469-471, 1998

29. Egger M, Davey Smith G, Schneider M and Minder C: Bias in meta-analysis detected by a simple, graphical test. BMJ 315: 629-634, 1997.

30. Tobias A, Saez M, Galan I and Campbell MJ: Sensitivity analysis of common statistical models used to study the short-term effects of air pollution on health. Int J Biometeorol 47: 227-229, 2003.

31. Hayes JD, Flanagan JU and Jowsey IR: Glutathione transferases. Annu Rev Pharmacol Toxicol 45: 51-88, 2005.

32. Sharma R, Yang Y, Sharma A, Awasthi S and Awasthi YC: Antioxidant role of glutathione S-transferases: Protection against oxidant toxicity and regulation of stress-mediated apoptosis. Antioxid Redox Signal 6: 289-300, 2004.

33. Strange RC, Lear JT and Fryer AA: Glutathione S-transferase polymorphisms: Influence on susceptibility to cancer. Chem Biol Interact 111-112: 351-364, 1998

34. Moaven O, Raziee HR, Sima HR, Ganji A, Malekzadeh R, A'Rabi A, Abdollahi A, Memar B, Sotoudeh M, Naseh H, et al: Interactions between glutathione-S-transferase M1, T1 and P1 polymorphisms and smoking, and increased susceptibility to esophageal squamous cell carcinoma. Cancer Epidemiol 34: 285-290, 2010.

35. Karami S, Boffetta P, Rothman N, Hung RJ, Stewart T, Zaridze D, Navritalova M, Mates D, Janout V, Kollarova H, et al: Renal cell carcinoma, occupational pesticide exposure and modification by glutathione S-transferase polymorphisms. Carcinogenesis 29: 1567-1571, 2008.

36. Sreeja L, Syamala V, Hariharan S, Syamala VS, Raveendran B, Sivanandan CD, Madhavan J and Ankathil R: Glutathione S-transferase M1, T1 and P1 polymorphisms: Susceptibility and outcome in lung cancer patients. J Exp Ther Oncol 7: 73-85, 2008. 